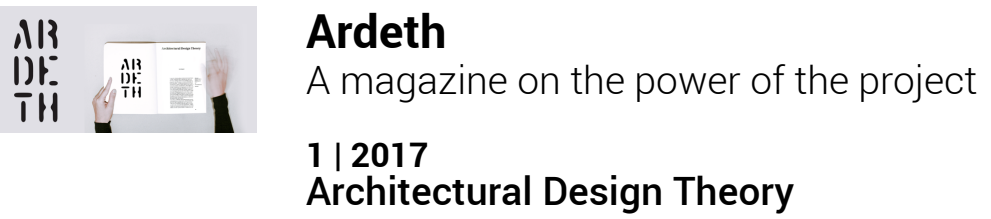

\title{
Il disegno, il General Intellect e la città: per una rilettura del Vasari
}

Franco Farinelli

\section{(2) OpenEdition}

Edizione digitale

URL: http://journals.openedition.org/ardeth/952

ISSN: 2611-934X

\section{Editore}

Rosenberg \& Sellier

\section{Edizione cartacea}

Data di pubblicazione: 1 octobre 2017

Paginazione: 49-60

ISSN: 2532-6457

Notizia bibliografica digitale

Franco Farinelli, « II disegno, il General Intellect e la città: per una rilettura del Vasari », Ardeth [Online],

1 | 2017, online dal 01 octobre 2017, consultato il 13 novembre 2020. URL : http://

journals.openedition.org/ardeth/952 


\section{Il disegno, il General Intellect e la città: per una rilettura del Vasari}

Franco Farinelli

Abstract

Starting from a reinterpretation of $16^{\text {th }}$ Century "teoriche" focusing on the notion of "disegno", the article examines the use of this terms in Giorgio Vasari's work. For the author of Le vite de' più eccellenti pittori, scultori e architettori (1550 and 1568), drawing ("disegno") corresponds to a form of "cognitive-instrumental rationality" that does not answer to a declared aim, but that is nonetheless able to construct the «space that the rationalization of images of the world in the West opens up for a modernization of society» (Habermas, 1986). The comparison between the two editions of the Vite (Torrentini, 1550 and Giunti, 1568) shows a fundamental change in paradigm: in the first edition every artist acquires exemplary value as an individual; whereas in the second edition the distinctive trait lies not in individual artists but rather in Florentine artistic canon, intended as universal and objective. In this shift, disegno becomes an explicitly intellectual device that allows to transfer the qualities of individuals to a collective intellect.
Affiliation

Università di

Bologna.

franco.farinelli@ unibo.it

DOI

10.17454/ARDETH01.04

ARDETH\#01 


\section{Il disegno e i disegni}

All'inizio della seconda metà del Cinquecento le Vite del Vasari testimoniano in maniera sottilmente eloquente, e ancora da intendere fino in fondo, la modalità e insieme l'irreversibilità del transito verso la città moderna della “città pellegrina” di Agostino, instabile perché programmaticamente in marcia verso la riunificazione del mondo terreno con quello celeste. Più precisamente, per Agostino (XVIII, 1 e 54) è la città di Dio a essere pellegrina all'interno della città di questo mondo il che fa di quest'ultima un'entità non soltanto estremamente dinamica ed eterogenea dal punto di vista della propria natura ma ancora munita di una struttura ricorsiva, come tutte le combinazioni in cui le cose stanno dentro altre cose. Di qui la sua estrema tensione interna, che dalla caduta dell'impero romano arriva alle soglie del Rinascimento, quando inizia l'epoca della città spaziale, che appunto in Vasari trova la sua prima forma di coscienza, implicita ma non per questo meno concreta ed efficace. Ed è il disegno il dispositivo da cui la produzione di tale forma dipende. Basterebbe al riguardo proprio l'insistenza, espressa all'inizio delle Vite, circa la similitudine tra il corpo e l'edificio, per cui «la facciata vuole avere decoro e maestà et essere compartita come la faccia dell'uomo» (Vasari 1, p. 19). Si tratta del richiamo al principio quattrocentesco, teorizzato dall'Alberti e dal Martini e tanto caro al Filarete (Rykwert 1996, pp. 56-68), dell'applicazione alla struttura architettonica e alle sue proporzioni del canone valido per quella umana: richiamo nel caso specifico rinforzato dalle ragioni della simmetria e della polemica contro la "maniera tedesca" a favore "dell'antica e moderna, vera e buona" (Vasari 4, p. 615). Ma proprio il tono precettistico-normativo, e il ricorso alle ragioni della simmetria, avverte qui dell'ormai quasi consumata rottura dell'antica, premoderna (perchè precedente ogni artificiale sguardo prospettico) solidarietà e omologia, anzi ontologica affinità, tra gli uomini e le cose, i primi ormai decisamente avviati a trasformarsi in soggetti e le seconde in oggetti privi di espressione: come se anche in questo caso il Vasari stesso non potesse sfuggire al destino de «gli scrittori [che] cominciorono a far memoria delle cose state innanzi a loro", i quali possono iniziare soltanto da ciò di cui ancora si riesce ad avere notizia, vale a dire appunto da quel che va sparendo (Vasari 2, p. 13). Nei cui confronti le «vivacissime e pietosissime penne delli scrittori» (Vasari 1, p. 9) hanno però ben altra funzione di quella di conservarne semplicemente il ricordo, a dispetto di quanto il Vasari vorrebbe far intendere. È con la retorica della scrittura, e con la rivendicazione della propria insufficienza, che le Vite s'inaugurano e terminano: all'inizio, nella dedica a Cosimo, sottolineando l'inadeguatezza della "penna d'un disegnatore" rispetto alla "verità della storia”, vale a dire la miseria di una scrittura che, "per essere incolta e così naturale com'io favello, non è degna de lo orecchio di Vostra Eccellenzia»; alla fine, ribadendo di aver "scritto come pittore", vale a dire come chi ha «più le mani ai pennelli che alla penna, e più il capo ai disegni che allo scrivere» (Vasari 1, p. 3; 6, p. 412). Tra i due estremi, 
dunque nel corso dell'intera opera, quel che però appunto si afferma è «il disegno, ch’è disegno nostro" (Vasari 1, p. 16), dove "nostro" non significa soltanto toscano cioè fiorentino ma (anche) del Vasari stesso. E proprio nell'ambiguità o bivalenza di tale riferimento, culturale e personale a un tempo, la sottigliezza vasariana inizia ad esprimersi, la sua astuzia a far capolino, a manifestarsi con una rapidissima strizzata d'occhio.

L'impalcatura ideale della struttura narrativa delle Vite, così come l'organizzazione concreta dei suoi contenuti, si regge su una triplice accezione del termine "disegno", e sull'intreccio dei corrispettivi livelli di riferimento: disegno mentale, disegno inteso come opera della mano, disegno inteso come oggetto fisico, come foglio di carta dotato di tracce (Faietti, 2011, pp. 13-37). Il disegno di cui Vasari rivendica qui la proprietà significa però ancora un'altra cosa, è intenzione, piano, programma, è l'espressione di un'autentica forma di "razionalità cognitivo-strumentale” volta a uno scopo non esplicitamente dichiarato, la cui decifrazione passa precisamente attraverso l'indicazione dello «spazio che la razionalizzazione delle immagini del mondo in Occidente apre per una modernizzazione della società» (Habermas, 1986, p. 953). Il suo accertamento insomma implica la verifica funzionale dei primi tre livelli che il disegno per Vasari significa, e mette capo alla matrice dell'idea di città che ancora oggi noi abbiamo in testa. Tutta o quasi l'arguzia del Vasari si condensa sul carattere sfuggente o addirittura equivoco del rapporto tra disegno e intelletto, problema principale della sua teorica. Il primo, "padre delle tre arti”, procede dal secondo, si afferma all'inizio, nel capitolo XV del primo libro dell'edizione giuntina. Ma all'attacco del Proemio delle vite che nella stessa edizione segue, si legge che il disegno «è l'istessa anima che concèpe e nutrisce in se medesima tutti i parti degli intelletti» (Vasari 1, p. $111 ; 2$, p. 3): dunque in questo caso si sosterrebbe il contrario, la precessione del disegno rispetto al complesso dei compiti intellettivi, o almeno la sua coincidenza nei confronti dell' intelletto. Si potrebbe osservare che l'intelletto, al singolare, non corrisponde necessariamente alla pluralità delle sue manifestazioni, e dunque potrebbe non esservi contraddizione: e si avrebbe ragione. Si esamini allora con attenzione e fino in fondo "Che cosa sia disegno" per Vasari.

\section{Il disegno e l'intelletto}

Appunto perché proviene dall'intelletto il disegno ricava dalla molteplicità dell'esistente un "giudizio universale" di tutte le cose della natura, e poiché da tale conoscenza nasce "un certo concetto o giudizio" si forma nella mente quella cosa che "espressa con le mani si chiama disegno", espressione visibile di un concetto che si ha nell'animo. E fin qui nessun problema. Ma subito dopo si aggiunge che la perfezione e l'eccellenza delle arti si manifestano se «l'intelletto manda fuori i concetti purgati e con giudizio", il che avviene soltanto quando «la mano sia mediante lo studio et essercizio di molti anni spedita et atta a disegnare et esprimere bene qualunque cosa ha la natura creato». Insomma:«l'idea non può 
vedere né imaginare perfettamente in sé stessa l'invenzioni, se non apre e non mostra il suo concetto agl'occhi corporali», perché soltanto «disegnando in carta, si viene a empiere la mente di bei concetti e s’impara a fare a mente tutte le cose della natura, senza averle a tenere sempre inanzi» (Vasari 1, p. 111; 6, pp. 156-157). In tal modo non vi sono dubbi: la qualità dell'intelletto dipende dall'esercizio del disegno, sicché non è possibile stabilire nessuna vera primazia dell'uno rispetto all'altro, nel senso che la logica del loro rapporto non è di natura lineare ma dichiaratamente ricorsiva. Come tra Tre e Quattrocento già s'avvisava: «sai che tt'avverrà praticando il disegniare di penna? Che tti farà sperto, pratico $\mathrm{e}$ capace di molto disegno entro la testa tua» (Cennini, 2003, p. 71). E come ha scritto Henri Focillon (1990, p. 134): «La mente fa la mano, la mano fa la mente».

Trattandosi di mani e ricorsività viene in mente, anche in questo caso, una celebre litografia, le Mani che disegnano di Escher: una mano che disegna un'altra mano che a sua volta, circolarmente, disegna la prima. Douglas Hofstadter (1984, pp. 747-748) ne ha svelato l'assunto: la riduzione del mondo a un disegno in grado di includere anche la mano dell'autore e dunque di cancellare la concreta esistenza di quest'ultima, confinata all'interno di un "livello inviolabile" all'occhio. Forse è proprio questa la ragione per cui la teorica vasariana non riesce a ricondurre il disegno a un rapporto definito nei confronti dell'intelletto: quel che di conseguenza le Mani che disegnano infatti rivelano (ed è perciò che fanno impressione) è l'autonomia del disegno rispetto al disegnatore, è la possibilità che il disegno stesso, ogni disegno, sia portatore di un'intenzione, di una volontà indipendente dalla mano dell'autore. Qui di seguito si tenta di mostrare come questo sia vero anche nel caso del Vasari, e quanto questo abbia a che fare con la moderna e contemporanea riduzione della civitas all'urbs, anzi del loro complesso alla semplice componente materiale, fino a risultare decisiva se non originaria. Sotto tal profilo, può essere fin d'ora anticipato, non vi è molta differenza tra quel che Vasari pensa dell'idea (la forma che è frutto del disegno) e l'idea che un secolo prima il cardinal Cusano (2003, p. 42) aveva della mente, immagine esemplare di tutte le cose che chiama all'essere dando loro forma, cioè misura. E così come per Cusano la mente era una mappa (Farinelli, 2009, p. 155) lo stesso vale per l'idea vasariana. Sicché va inteso con estremo giudizio quel che Vasari (3, p. 3) dice dove protesta che se avesse voluto limitarsi a redigere l'inventario degli artisti, delle loro patrie e delle loro opere l'avrebbe «potuto fare con una semplice tavola, senza interporre in parte alcuna il giudizio» suo. In realtà le sue Vite, cioè la sue storie, sono la formidabile animazione di una tavola, la messa in scena del suo dettato, la sua versione allegorica, proprio secondo la definizione che dell'allegoria vale per la retorica classica, quella di un pensiero sotto l'immagine di un altro pensiero, che serve a rendere il primo "più sensibile e più evidente che se fosse presentato direttamente e senza nessuna specie di velo" (Fontanier, 1977, p. 114). 
Vale insomma per Vasari proprio quel che egli stesso scrive: è vero che «il buon giudizio e l'occhio più giuoca in tutte le cose che non fa la misura delle seste», cioè del compasso; ma è anche vero, come testimonia nientemeno Michelangelo, che bisogna «avere le seste negli occhi e non in mano", appunto "perché le mani operano e l'occhio giudica» (Vasari 4, p. 236; 6, p. 109). Come dire, insomma, che l'ideale consiste in un giudizio che fin dall'inizio incorpori la misura, vale a dire la logica della tavola, come ancora fino a tutto l' Ottocento la mappa sarà chiamata. Ma di quale entità l'idea del Vasari è la mappa, di quale mente essa è la forma? Si può intanto rispondere con le parole che Francesco Medici, allora principe di Firenze, risponde al Vasari che gli mostra la sua "invenzione” per adornare la «volta di dentro di quella superba e meravigliosa fabbrica della cupola condotta per opera et arte di quel raro e pellegrino ingegno di Filippo di ser Brunellesco». Francesco riconosce che «è un disegno molto bello e non è cosa che a uno sguardo solo» ci si «possa saziare: ha di bisogno di matura e particolare considerazione» (Vasari, 2007, p. 237).

\section{Il disegno e le Vite}

Con tali parole si chiudono i Ragionamenti di Palazzo Vecchio, la guida alle pitture che l'adornano che Vasari inizia a scrivere nel 1558, dunque quattro anni dopo la sua presa di servizio agli ordini di Cosimo, e tra la prima e la seconda stesura delle Vite. Da tempo la critica ha messo in risalto la differenza di registro tra le due edizioni: «quello assoluto e unitario del 1550, e quello discontinuo e narrativo del 1568», al cui interno l'opera d'arte non risulta più semplicemente legata al "sottile filo biografico", ma molto più contestualizzata rispetto all'articolazione ambientale e alla trama della committenza, fruizione e divulgazione (Barocchi, 1984, pp. 87, 162, 159). Indubbiamente è così: di sicuro nell'ultima edizione, la Giuntina, si riserva «rispetto alle persone, a’ tempi et ai luoghi» (Vasari 3, p. 274) più che nella Torrentiniana. Ma non è soltanto così. Si prenda ad esempio la vita di Lorenzo Ghiberti, e si faccia il paragone tra le due versioni. Si spiega, all'inizio della prima, come il «santissimo lume d'esempio a molti» costituito da coloro che acquistano fama inciti i concittadini al "sudore della virtù", in funzione degli onori, della ricchezza, dei trionfi, delle "imagini”, vale a dire delle opere d'arte celebrative; «e da questo nasceva negli antichi che le città in bellezza si mantenevano per giustamente guiderdonare coloro che se medesimi e le loro patrie onoravano». In altri termini: l'emulazione dei cittadini si traduceva nello splendore urbano, inteso come elemento di ricompensa e segno del valore degli abitanti. Nella Giuntina non è più così, della connessione e dello scambio tra gli uomini e le pietre non si fa più parola, e al posto dell'interrelazione tra civitas e urbs, del rapporto costitutivo in senso civico tra le cose e le persone, fa la propria apparizione "l'invidia" che quasi sempre perseguita la virtù, subentra il semplice agire interpersonale. Così, a proposito del celebre episodio delle porte del battistero di San Giovanni, nell'edizione del 1550 Lorenzo viene riconosciuto superiore "nel getto” da Donatello e Brunelleschi «per mostrar l'amore, 
che prima a se stesso, poi alla sua patria portava»: notazione che puntualmente scompare nella versione stampata nel 1568 (Vasari 3, pp. 75-76). E a farvi caso quando nella Giuntina interviene una connessione tra il soggetto e l'oggetto, quest'ultimo si configura sempre e soltanto secondo due varianti: o un'opera d'arte, come per esempio nel caso della vita di Raffaello, che dopo la frequentazione della pittura fiorentina variò ed abbellì molto la sua maniera (Vasari 4, p. 62); oppure, come nel proemio alla vita del Perugino assume la forma più indefinita e la minor consistenza che si possa pensare, la forma anzi di un autentico non-oggetto, coincidendo con l'impalpabile aria fiorentina che rende "gli ingegni liberi di natura». E il Vasari non potrebbe, nell'occasione, essere più eloquente e significativo, perché dopo aver ricordato che tali ingegni debbono, per divenire ricchi, partirsi dal capoluogo toscano per «vender fuora la bontà dell'opere sue, e la riputazione di essa città, come fanno i dottori quella del loro Studio», così di seguito conclude: «Perché Firenze fa degli artefici suoi quel che il tempo delle sue cose, che, fatte, se le disfà et se le consuma a poco a poco» (Vasari 3, 85). Per Paola Barocchi (1984, p. 118) il brano in questione, già presente nella Torrentiniana, vale a illustrare la natura del "fiorentinismo" del Vasari, almeno prima del suo ritorno da Roma a Firenze agli ordini di Cosimo: un'affezione venata da una specie di rammarico, quasi di un senso di esilio, dunque comprensibilmente riferita alle sue personali vicende. Ma la chiusa significa molto di più, oltre l'ineluttabilità del tempo che passa e la conseguente allusione alla natura irredimibile del singolo percorso esistenziale, dice anzi esattamente il contrario: afferma che a Firenze gli artefici, cioè le persone, subiscono lo stesso destino riservato nel corso del tempo alle cose, che a poco a poco si consumano, e di cui perciò si finisce con il perdere memoria. Invece di commuoversi sul carattere effimero dell'esistenza umana, bisogna qui porre mente all'analogia, anzi alla funzione ontologica dell'omologia tra i soggetti e gli oggetti, per cui i primi si trasformano esemplarmente nei secondi, proprio secondo il canone della regola prospettica moderna, per cui chi guarda secondo la "dolce prospettiva” in realtà finisce sempre con il guardare l’occhio di Medusa, che lo pietrifica e lo cosifica. Al riguardo l'espressione vasariana non potrebbe essere più precisa e perspicua, a segno e come effetto dell'intervenuto, secolare lavorio del modo di regolazione spaziale di marca fiorentina. E allo stesso tempo essa consente di intendere meglio il paradossale processo che conduce all'affermazione di quest'ultimo. L'agostiniana “città pellegrina” eliminava al massimo il divario tra le cose e gli esseri viventi, nel senso dell'equivalenza di quelle con questi. Al contrario soltanto a patto della preliminare, inversa riduzione dei soggetti agli oggetti che sotto il portico degli Innocenti per la prima volta si produce diventa paradossalmente pensabile la loro reciproca differenza, secondo quel "pensiero oggettivo" che proprio in tal modo e in tale luogo nasce, e che sulla base dell'esperienza effettiva conosce appunto soltanto nozioni alternative, «concetti puri che si escludono» (Merleau-Ponty 1965, pp. 86-87) come senza l'avvento dell'intervallo di natura prospettico-spaziale non potrebbero fare. 
E tale dicotomico regime cognitivo si traduce, nella seconda stesura delle Vite, nella minor enfasi riservata al rapporto tra l'artista e la sua patria, come per esempio nel caso dell'aretino Parri Spinelli o anche del fiorentino Donatello (Vasari 3, pp. 113 e 202) . Ma soprattutto il Vasari mediceo opera una netta separazione tra civitas e urbs. Soltanto al signore dell'universo, a Cosimo, è concesso il privilegio di tenerle davvero insieme, di vivere ancora all'interno di una città in cui tra le pietre e gli esseri viventi non esiste nessuna ontologica frattura, nel senso che gli oggetti risultano proiezione (com'è proprio il caso di dire) del soggetto: Cosimo infatti «non perdona né a spesa né a cosa veruna perché tutte le fortificazioni et edificii publici et privati corrispondino alla grandezza del suo animo», e siano «non meno belli che utili, né meno utili che belli» (Vasari 4, p. 243). Altrimenti la civitas si riduce, specie nell'ultima edizione, al rapporto interno alla cerchia degli artisti e tra questi e i committenti. Proprio in tale rapporto consiste anzi "l'aria" fiorentina appena ricordata, come espressamente e fuor d'ogni dubbio si dichiara all'inizio della vita di Gaddo Gaddi, amico di Cimabue (Vasari 2, pp. 81-82). Così appunto Brunelleschi e Donatello riconoscono, anche se non tenuti e contro il proprio interesse, la maggior bravura del Ghiberti. E allo stesso modo si amano «fraternamente l'un l'altro, godendo ciascuno così dell'onor e pregio dell'amico come del proprio» Simone Memmi e Taddeo Gaddi, ambedue allievi di Giotto, Mariotto Albertinelli e Baccio dalla Porta «che erono un'anima et un corpo", e si potrebbe continuare nell'elenco, fino ai membri della cerchia raccolta intorno a Raffaello "che stavano uniti e di concordia tale che tutti i mali umori nel veder lui si amorzavano, et ogni vile e basso pensiero cadeva loro di mente» (Vasari 2, p. 211; 4, pp. 105, 221-222).

\section{Il disegno, il segreto, la verità}

L'ultimo sostantivo non va sottovalutato, poiché riassume in maniera precisa e allo stesso tempo adeguatamente ampia la reale natura, cognitiva e insieme sociale, del problema in questione. Esattamente come il disegno, anche la messa in comune (il "conferire”) delle difficoltà del lavoro artistico, cioè il reciproco atteggiamento caritatevole degli artefici, produce concetti "purgati”, come alla lettera la natura del rapporto tra Gaddo e Cimabue, nel brano appena richiamato testimonia. Di conseguenza il disegno, che nella teorica e nel Proemio corre il rischio di essere inteso come espressione di una singola mano, di un intelletto singolare, di un'anima solitaria, qui assume la natura di un vero e proprio intelletto generale, di un "general intellect” nel senso che Marx (1976, pp. 716 e ss.) assegna al termine nel celebre frammento sulle macchine. La «natura non costruisce macchine, locomotive, ferrovie, telegrafi elettrici, telai meccanici...", nota Marx. E prima ancora nemmeno produce disegni, vien da aggiungere, tanto più che non soltanto senza di essi nessuna di quelle macchine può essere costruita, ma il disegno stesso è la prima macchina, l'originaria machina machinarum: almeno da Turing in poi infatti, perciò da quasi un secolo ormai, una macchina è nient'altro che una 
tavola che contiene dei simboli (Iorio Giannoli, 2003, pp. 29-30), dunque in una sola parola una mappa, il dispositivo che rappresenta le cose e, insieme con esse, l'astratta nozione di calcolo (che in assenza della tavola non si darebbe) di cui quest’ultime sono espressione. Il disegno cioè la tavola è alla lettera, proprio come il Vasari lo intende e per riprendere Marx, il primo degli «organi dell'intelligenza umana creati dalla mano umana», il primo agente della "potenza materializzata del sapere», il primo strumento che «mostra in quale misura il sapere sociale generale, la conoscenza, si è trasformato in forza produttiva immediata, e quindi fino a che punto le condizioni del processo vitale stesso della società sono passate sotto il controllo dell'intelligenza generale e rimodellate in accordo con essa".

I confini della messa in comune e della condivisione di tale intelletto sociale sono appunto quelli della civitas degli artefici e dei loro patroni. Attraverso l'attività artistica l'intelligenza che crea la ricchezza (il "general intellect” nel senso proprio, marxiano, del termine) e l'intelligenza che dalla ricchezza è creata stabiliscono un comune perimetro, si riconoscono all'interno della stessa comunità: quella dei networkers, di coloro che, all'interno dell'organizzazione del lavoro sono dotati di effettivo potere discrezionale, accesso all'informazione e, conseguentemente, di capacità di generare sapere stabilendo connessioni di propria iniziativa, e che perciò risultano distinti dai networked (che non possono decidere come, quando, perché e con chi lavorare) e dagli switched-off, capaci soltanto di mansioni limitate e scarsamente interattive perché definite da istruzioni univoche (Castells, 1996, pp. 321-322). In comune tutti costoro hanno un'urbs, una città composta d'«onorate fabriche e [d']utili e vaghi componimenti di storie» (Vasari 2, p. 179) che svolgono il ruolo di garantire la connessione con la civitas, e in questo consiste la loro specifica funzione politica, relativa al sapere della pólis: ecco perché Pier Soderini, il gonfaloniere di Firenze, si sente in dovere di giudicare, come del resto gli altri cittadini ma in modo più plateale e ostentato, la lunghezza del naso della statua di Michelangelo (Vasari 6, p. 53). Si tratta in ogni caso di elementi isolati, limitati, discreti, oggettivi proprio perché individuati in virtù di uno sguardo prospetticamente addestrato, dunque sistematicamente votato alla scomposizione della struttura urbana o in senso puntuale o in senso lineare. E sotto tal profilo, in relazione cioè a tale processo, il passaggio dall'edizione torrentiniana alla giuntina si caratterizza in termini decisamente coerenti e progressivi. Per convincersene basta comparare per esempio le due versioni della vita di Andrea del Sarto, dove il giudizio sulle sue disavventure affettive sono subito sostituite da quello sulle sue figure e sull'aria delle sue teste: se nell'edizione del 1550 ogni artista acquistava, con la propria vita, valore esemplare rispetto alle possibilità generali, svolgeva cioè in termini locali e soggettivi una vicenda universale, nel testo edito nel 1568 il racconto delle vicissitudini umane appare molto più decisamente piegato all'imposizione dell'esemplarità, vale a dire dell'universalità e dell'oggettività, del canone artistico fiorentino 
(Vasari 4, pp. 341-342). Presupposto di tale operazione, che investe seppure con accenti diversi ambedue le redazioni, è appunto la riduzione della struttura urbana a immagine, cioè a disegno, «capo necessario di tutte [le arti], e non l'avendo, non si ha nulla». Infatti «se bene tutti i segreti et i modi sono buoni, quello è ottimo per lo quale ogni cosa perduta si ritrova, et ogni difficil cosa per esso diventa facile». Un segreto dunque, che consente di compiere anche le cose "sopraumane" (Vasari 1, p. 172), circa il quale si fornisce qualche concreta indicazione all'inizio della vita di Baccio d'Agnolo, asceso dal basso dal suo mestiere d'intagliatore e fabbricante di modelli in legno alla professione d'architetto: passaggio reso possibile dal fatto che chi maneggia di continuo le colonne, le cornici, i basamenti e gli altri elementi di cui un edificio si compone finisce per apprenderne, indipendentemente dalle loro dimensioni, le reciproche misure ovvero proporzioni, dal cui complesso si tira proprio "l'ordine e l'architettura” che giusto nel disegno si depositano. In ciò consiste appunto il segreto di quest'ultimo, nel consentire di mettere in relazione le singole parti con il complesso, e di risalire se del caso da quelle a questo e viceversa, dal particolare al generale e al contrario, secondo le mosse (indifferentemente, cioè nella doppia direzione) dell'induzione e della deduzione: al punto che la vista di una sola unghia di un leone scolpita in un masso consente una cosa davvero portentosa, comprendere «con l'intelletto da quella misura e forma le parti di tutto l'animale e dopo il tutto insieme», come ad averlo "presente e dinanzi agl'occhi» (Vasari 4, pp. 609-610; 1, 111).

Proprio secondo quel che avviene sotto il portico degli Innocenti l'immateriale diventa così materiale e il materiale immateriale, come se rifluendo dal mondo, cioè dal quotidiano e diretto commercio tra gli uomini e le cose, ogni possibilità di trasmutazione di questo in quello e viceversa si depositasse nei segni tracciati in piano, strumenti esclusivi della mediazione. Il che fa del disegno un genuino dispositivo ontologico, un autentico commutatore dei livelli di realtà, attivato dalla presenza di un semplice indizio, di un minuscolo brano, di un puntuale riferimento. E all'inverso ogni isolato frammento, ogni decontestualizzata particella implica (vale a dire di necessità rimanda a) un contestuale insieme, un sistematico ordito, un impercettibile eppure esistente complesso, che proprio nella sua invisibilità, nel suo sottrarsi a qualsiasi evidente manifestazione diventa implicita intenzione, piano appunto, metamodello. In una sola parola: un "ordine” proprio, non soltanto nel senso architettonico di "proporzioni unite e di corpi» (Vasari 1, p. 15) fondato sull'individuazione e la compatibilità tra funzioni e usi diversi dello spazio (Conforti, 2011, p. 200) ma anche in quello, contiguo e affine, di imposizione, prescrizione, selezione, dunque nel senso del comando del signore, della decisione politica. È per questo, in fin dei conti, che Vasari ritiene il disegno «un modo di trattare col mondo che gode un accesso privilegiato alla verità» (Williams, 2010, p. 69): perché esso è in grado di riassumere ed esplicare non soltanto la logica dei processi cognitivi fondamentali ma 
anche la logica del potere, esso è l'attività in cui questa e quella visibilmente finiscono con il coincidere, rivelano la propria comune matrice, che soltanto l'artista e il signore padroneggiano. Ne consegue che come il signore anche l'artista comanda. E se il comando di Cosimo cessa con la sua morte, quello del Vasari dura fino ai giorni nostri e oltrepassa gli stessi confini della modernità, se è vero che il postmodernismo si fonda prima di tutto sulla consapevolezza che «i contenuti altro non sono che ancora più immagini», e che esso è quel che resta quando «il processo della modernizzazione è completato e la natura è sparita per sempre» (Jameson, 1991, p. IX).

È sufficiente aprire qualsiasi guida turistica di qualsiasi città per rendersene conto: operativamente, e perciò concretamente, Bangkok, Lione, Helsinki e tutte le altre città del mondo sono ridotte sulle guide, silenziosamente ma sistematicamente, a un'unica città, sulla base di un minimo comun denominatore che è quello fiorentino, nel senso che ognuna di esse viene funzionalmente ma invariabilmente scomposta secondo le regole programmatiche della prospettica sintassi urbana di Firenze, impostata su punti (i monumenti, le discrete perché puntiformi opere dotate di una particolare cifra artistica) e linee rette (le strade che assicurano il più rapido spostamento da un punto all'altro, che a Firenze sono di fatto più diritte che altrove, riescono esemplarmente rettilinee). In altri termini: sotto il pretesto di narrare le vite degli artisti fiorentini, e letteralmente inventando quel che oggi viene chiamato il marketing territoriale, il Vasari afferma il modello urbano fiorentino come l'unico possibile, attraverso una sofisticatissima strategia cui qui si può soltanto accennare, fondata su due principî fondamentali. Il primo consiste nell'equivalenza tra Firenze, Palazzo Vecchio e le Vite stesse. Il secondo nell'accorgimento di imporre una visione statica della realtà (il modello prospettico) attraverso il ricorso a quella che, nella sua genealogia della mente del viaggiatore, Eric J. Leed (2000, p. 100) chiama la logica dell'«ordine progressivo del transito egoistico»: astuzia con cui il Vasari riesce a far passare un ordinamento categoriale e selettivo dei dati per il suo contrario, per la semplice successione sequenziale del loro flusso (Bateson, 1951, pp. 184-186), riuscendo per tal via a far sembrare vivente lo spazio, cioè «un mito, un fantasma, una finzione della geometria», qualcosa che non ha nulla a che fare con la percezione (Gibson, 1999, p. 37). Come è stato spiegato: far vedere è un atto filosofico e insieme politico, perché costruisce lo sguardo di un soggetto che parla «nello spazio al cui interno entra in relazione con altri soggetti parlanti» (Mondzain, 2003).

È il Vasari stesso a sottolineare tale aspetto della propria strategia: al giovane principe Francesco che gli fa notare, durante la sua visita a Palazzo Vecchio, la discrasia tra l'ordine delle pitture e l'ordine del discorso con il quale l'autore le va descrivendo, egli riconosce appunto di essersi lasciato trasportare «nel dire la continuazion delle storie dipinte più che l'ordine de’ significati» (Vasari, 2007, p. 34). È appunto la dimensione diacronica che il Vasari vuol far risaltare, non quella sincronica, come il figlio del 
grande Cosimo, specie di prototipo del turista contemporaneo, non può fare a meno di notare, lamentando la carenza dell'illustrazione verbale, l'assenza della spiegazione di certe figure. E per Vasari Palazzo Vecchio e Firenze sono la stessa cosa, proprio in funzione dell'affermazione del potere mediceo secondo un'autentica forma politica. Anche questo, nei Ragionamenti, è detto espressamente ed esemplarmente. Così come subito dopo si aggiunge che «la poesia e la pittura usano come sorelle i medesimi termini» (Vasari, 2007, pp. 10-11 e 13), vale a dire che quest'ultima, che per Vasari inizia dal disegno, corrisponde a un testo (Le Mollé, 2007, p. XXV). Ed è per questa ragione, vien da supporre, che l'edizione giuntina si chiude con la minuta descrizione degli apparati scenografici e allegorici allestiti nel 1536 in occasione delle nozze di Margherita d'Austria con Alessandro de’ Medici: per mano del Vasari Firenze in tal modo diventa, come più tardi Palazzo Vecchio, un'unica successione di distinte pitture e sculture, non soltanto ribadendo la continuità e coerenza, all'interno del progetto vasariano e per mezzo della mediazione pittorica, tra il testo delle Vite e la struttura fiorentina, ma anche per tal verso significando e ribadendo, con l'identità tra città e insieme di puntiformi opere d'arte, il primato del modello fiorentino su ogni altro modello urbano, civile e politico. Quel che era poi l'autentico “disegno interno”, come avrebbe detto Michelangelo, di tutta l'opera del Vasari: non soltanto il «mitografo del potere assoluto» (Muccini e Cecchi, 1995, p. 5), ma anche quello della città assoluta, cioè della città prospettico-spaziale moderna.

\section{Bibliografia}

Agostino (2002), La città di Dio, Milano, Bompiani.

Barocchi, P. (1984), Studi Vasariani, Torino, Einaudi.

Bateson, G. (1951), Information and Codification. A Philosophical Approach, in J. Ruesch, G. Bateson, Communication. The Social Matrix of Psychiatry, New York, Norton.

Castells, M. (1996), La nascita della società in rete, Milano, Bocconi.

Cennini, C. (2003), Il libro dell'arte, a cura di F. Frezzato, Vicenza, Neri Pozza. Conforti, C. (2011), “Ordine et disegno, disegno et ordine”: la Fabbrica degli Uffizi, in Id. (a cura di), Vasari, gli Uffizi e il Duca, catalogo della mostra omonima, Firenze, Giunti.

Cusano, N. (2003), I dialoghi dell'Idiota. Libri Quattro, a cura di G. Federici Vescovini, Firenze, Olschki.

Eck, D. (1982), Banaras: City of Light, Princeton, Princeton University Press.

Faietti, M. (2011), Il disegno padre delle arti, i disegni degli artisti, il disegno delle "Vite". Intersecazioni semantiche in Vasari scrittore, in M. Faietti, A. Griggo, G. Marini (a cura di), Figure Memorie spazio. La grafica del Quattrocento: appunti di teoria, conoscenza e gusto, Firenze, Giunti.

Farinelli. F. (2009), La crisi della ragione cartografica, Torino, Einaudi.

Focillon, H. (1990), Elogio della mano, in Id., Vita delle forme seguito da Elogio della mano, Torino, Einaudi.

Fontanier, P. (1977), Les figures du discours, Paris , Flammarion. 
Gauchet, M. (1985), Le désenchantement du monde. Une histoire politique de la religion, Paris, Gallimard.

Gibson, J.J. (1999), Un approccio ecologico alla percezione visiva, Bologna, il Mulino. Habermas, J. (1986), Teoria dell'agire comunicativo, II, Bologna, il Mulino.

Hofstadter, D. (1984), Gödel, Escher, Bach: un'eterna ghirlanda brillante, Milano, Adelphi.

Iorio Giannoli, G. (2003), Materializzazioni dell'anima. Dai modelli dell'intelligenza all'intelletto sociale, Roma, Manifestolibri.

Jaeger, W. (1954), Paideia. La formazione dell'uomo greco, II, Firenze, La Nuova Italia.

Jameson, F. (1991), Postmodernism, or, The Cultural Logic of Late Capitalism, Durham, Duke University Press.

Jaspers, K. (1965), Origine e senso della storia, Milano, Edizioni di Comunità.

Leed, E.J. (2000), La mente del viaggiatore. Dall'Odissea al turismo globale, Bologna, il Mulino.

Le Mollé, R. (2007), Vasari face à un grand projet, introduzione a G. Vasari, Ragionamenti di Palazzo Vecchio, Paris, Les Belles Lettres.

Marx, C. (1976), Lineamenti fondamentali di critica dell'economia politica, Torino, Einaudi.

Merleau-Ponty, M. (1965), Fenomenologia della percezione, Milano, il Saggiatore. Mondzain, M.-J. (2003), Le commerce des regards, Paris, Seuil.

Muccini, U. e Cecchi, A. (1995), Le stanze del Principe in Palazzo Vecchio, Firenze, Le Lettere.

Rykwert. J. (1996), The Dancing Column. On Order in Architecture, Cambridge (MA) - London, The MIT Press.

Sassen, S. (2008), Territorio, autorità, diritti. Assemblaggi dal Medioevo all'età globale, Milano, Bruno Mondadori.

Tasso, T. (1875), Prose diverse nuovamente raccolte ed emendate, I, a cura di C. Guasti, Firenze, Le Monnier.

Vasari, G. (1966-1987) Le vite de' più eccellenti pittori, scultori e architettori nelle redazioni del 1550 e 1568, ed. Bettarini-Barocchi, 8 voll., Firenze, Sansoni.

Vasari, G. (2007), Ragionamenti di Palazzo Vecchio, Paris, Les Belles Lettres.

Williams, R. (2010), La fenomenologia della virtù, in K. Burzer, C. Davis, S. Feser, A. Nava (a cura di), Le Vite del Vasari. Genesi, topoi, ricezione / Die Vite Vasaris. Entstehung, Topoi, Rezeption, Venezia, Marsilio. 